\title{
A GENERALIZATION OF STEINER'S FORMULAE
}

\author{
E. VIDAL ABASCAL
}

Let $C$ be an arbitrary convex curve in the plane of length $L$ and area $F$; and let $C_{\rho}$ be a curve parallel to $C$ at a distance $\rho$ from it, of length $L_{\rho}$ and area $F_{\rho}$. Then according to Steiner's classical result:

$$
L_{\rho}=L+2 \pi \rho, \quad F_{\rho}=F+\rho L+\pi \rho^{2} .
$$

In this paper we develop a generalization of these formulae for curves lying on a curved surface whose curvature $K\left(v^{1}, v^{2}\right)$ (referred to geodesic parallel coordinates) is a function of $v^{2}$ alone. Explicit formulae are derived in the case of surfaces of constant curvature. In this treatment it is necessary to put certain restrictions on the curve $C$ and the distance $\rho$ to replace Steiner's assumption of convexity. These restrictions (which are discussed below) are stated in their most obvious form, and a discussion of methods of relaxing them is deferred to a later paper. Our chief results are contained in the formulae (12) and (15) below.

Let the curve $C$ be a simple, closed, bounding, and differentiable curve on the surface $S$. Choose a coordinate system in which $v^{1}=0$ is the curve $C$, and in which $v^{2}=$ constant are the geodesics orthogonal to $C$. Further let $v^{2}$ be the arc length of $C$ measured positively for motion on the curve which keeps the bounded area to the left, and let $v^{1}$ be the arc length of geodesics normal to $C$ measured positively outward from $C$. Choose the unit normals to $C$ so that they point toward the interior of $C$. Then we have:

$$
d s^{2}=\left(d v^{1}\right)^{2}+g_{22}\left(v^{1}, v^{2}\right)\left(d v^{2}\right)^{2} ; \quad g_{22}\left(0, v^{2}\right)=1 .
$$

For the moment we ignore the question of determining the region of $S$ within which such a coordinate system is valid, and proceed to compute $\left(g_{22}\right)^{1 / 2}$. In this coordinate system we have the following relations (see L. P. Eisenhart An introduction to differential geometry, pp. 181 and 188)

$$
\begin{aligned}
& \frac{\partial^{2}\left(g_{22}\right)^{1 / 2}}{\partial v^{1} \partial v^{1}}+K\left(g_{22}\right)^{1 / 2}=0, \\
& \kappa_{g}\left(v^{2}\right)=\left[\frac{\partial\left(g_{22}\right)^{1 / 2}}{\partial v^{\prime}}\right]_{v^{1}=0},
\end{aligned}
$$

Received by the editors December 26, 1946. 
where $\kappa_{g}$ is the geodesic curvature of $C$.

We assume that $K$ is a differentiable function of $v^{2}$, that it is independent of $v^{1}$, and that it is never zero. Then the integration of (2) gives:

$$
\left(g_{22}\right)^{1 / 2}=f\left(v^{2}\right) \sin \left[v^{1}\left(K\left(v^{2}\right)\right)^{1 / 2}\right]+h\left(v^{2}\right) \cos \left[v^{1}\left(K\left(v^{2}\right)\right)^{1 / 2}\right] .
$$

If $K\left(v^{2}\right)$ is negative, complex numbers are introduced, and $f$ and $h$ must be so chosen that the resulting value of $\left(g_{22}\right)^{1 / 2}$ is real. From (3) we find that

$$
\begin{aligned}
\kappa_{g}\left(v^{2}\right)= & \left\{f\left(v^{2}\right)\left(K\left(v^{2}\right)\right)^{1 / 2} \cos \left[v^{1}\left(K\left(v^{2}\right)\right)^{1 / 2}\right]\right. \\
& \left.-h\left(v^{2}\right)\left(K\left(v^{2}\right)\right)^{1 / 2} \sin \left[v^{1}\left(K\left(v^{2}\right)\right)^{1 / 2}\right]\right\}_{v^{1}=0}
\end{aligned}
$$

or $\kappa_{g}\left(v^{2}\right)=f\left(v^{2}\right)\left(K\left(v^{2}\right)\right)^{1 / 2}$. Hence

$$
f\left(v^{2}\right)=\frac{\kappa_{g}\left(v^{2}\right)}{\left(K\left(v^{2}\right)\right)^{1 / 2}} .
$$

Furthermore equation (4) must be valid along $C$, on which $v^{1}=0$ and $\left(g_{22}\right)^{1 / 2}=1$. Therefore from $(4), h\left(v^{2}\right)=1$. Hence:

$$
\left(g_{22}\right)^{1 / 2}=\frac{\kappa_{g}\left(v^{2}\right)}{\left(K\left(v^{2}\right)\right)^{1 / 2}} \sin \left[v^{1}\left(K\left(v^{2}\right)\right)^{1 / 2}\right]+\cos \left[v^{1}\left(K\left(v^{2}\right)\right)^{1 / 2}\right] .
$$

The chosen coordinate system will fail to be valid whenever (1) $\left(g_{22}\right)^{1 / 2} \leqq 0$; or (2) when $v^{1}$ is so large that the region described overlaps itself. The second difficulty may be overcome by considering overlapping portions to be on separate covering sheets of $S$ (as in a Riemann surface), but we must assume that $\left(g_{22}\right)^{1 / 2}>0$. We let $C_{\rho}$ be the closed curve $v^{1}=\rho$ (const.) and restrict ourselves to the interior of $C_{\rho}$. Hence we require that:

$$
\frac{\kappa_{g}\left(v^{2}\right)}{\left(K\left(v^{2}\right)\right)^{1 / 2}} \sin \left[v^{1}\left(K\left(v^{2}\right)\right)^{1 / 2}\right]+\cos \left[v^{1}\left(K\left(v^{2}\right)\right)^{1 / 2}\right]>0
$$

for all $v^{2}$ and for $0 \leqq v^{1} \leqq \rho$. Without further assumptions on $K, \kappa_{g}$, and $\rho$ no simplification of (8) is possible. However, for constant $K$ the validity of (8) may be inferred from other simple assumptions as follows:

Case 1. $K=$ constant $>0$. Then if $\kappa_{q}\left(v^{2}\right)>0$ and $0 \leqq \rho \leqq \pi 2 K^{1 / 2}$ each term of (8) is positive, so (8) holds.

Case 2. $K=$ constant $<0$. Then (8) can more properly be written:

$$
\frac{\kappa_{g}\left(v^{2}\right)}{(-K)^{1 / 2}} \sinh \left[v^{1}(-K)^{1 / 2}\right]+\cosh \left[v^{1}(-K)^{1 / 2}\right]>0 .
$$


And here if $\kappa_{g}\left(v^{2}\right)>0$ and $\rho \geqq 0$, the inequality is valid.

These are the assumptions which correspond to Steiner's requirement that $C$ be convex, and henceforth we consider only curves $C$ and values of $\rho$ for which they are verified.

Then from ( 7$)$ the length of $C_{\rho}$ is given by:

$$
L_{\rho}=\int_{C}\left(g_{22}\left(\rho, v^{2}\right)\right)^{1 / 2} d v^{2}
$$

or

(10) $\quad L_{\rho}=\int_{C} \frac{\kappa_{g}\left(v^{2}\right)}{\left(K\left(v^{2}\right)\right)^{1 / 2}} \sin \left[\rho\left(K\left(v^{2}\right)\right)^{1 / 2}\right] d v^{2}+\int_{C} \cos \left[\rho\left(K\left(v^{2}\right)\right)^{1 / 2}\right] d v^{2}$.

(We note that (10) holds even if $C$ does not bound. However, the assumption that $C$ bounds is essential for further developments.)

When $K$ is constant, (10) may be simplified by the use of the Gauss-Bonnet formula:

$$
\int_{C} \kappa_{g}\left(v^{2}\right) d v^{2}=2 \pi-K \iint_{\text {Interior of } C}\left(g_{22}\right)^{1 / 2} d v^{1} d v^{2}=2 \pi-K F .
$$

Hence

$$
L_{\rho}=2 \pi \frac{\sin \left[\rho K^{1 / 2}\right]}{K^{1 / 2}}-F K^{1 / 2} \sin \left[\rho K^{1 / 2}\right]+L \cos \left[\rho K^{1 / 2}\right] .
$$

When $K$ is negative (12) may more appropriately be written:

$$
\begin{aligned}
L_{\rho}= & 2 \pi \frac{\sinh \left[\rho(-K)^{1 / 2}\right]}{(-K)^{1 / 2}}+F(-K)^{1 / 2} \sinh \left[\rho(-K)^{1 / 2}\right] \\
& +L \cosh \left[\rho(-K)^{1 / 2}\right] .
\end{aligned}
$$

We note that as $K \rightarrow 0,(12)$ and $\left(12^{\prime}\right)$ approach Steiner's formula. Finally to find $F_{\rho}$, the area of $C_{\rho}$, we consider

$$
F_{\rho}=F+\int_{C}\left\{\int_{0}^{\rho}\left(g_{22}\left(v^{1}, v^{2}\right)\right)^{1 / 2} d v^{1}\right\} d v^{2}
$$

or

$$
\begin{aligned}
F_{\rho}=F & +\int_{C}\left\{\int_{0}^{\rho} \kappa_{y}\left(v^{2}\right) \frac{\sin \left[v^{1}\left(K\left(v^{2}\right)\right)^{1 / 2}\right]}{\left(K\left(v^{2}\right)\right)^{1 / 2}} d v^{1}\right\} d v^{2} \\
& +\int_{C}\left\{\int_{0}^{\rho} \cos \left[v^{1}\left(K\left(v^{2}\right)\right)^{1 / 2}\right] d v^{1}\right\} d v^{2}
\end{aligned}
$$


When $K$ is constant, (14) simplifies as follows owing to (11):

(15) $F_{\rho}=L \frac{\sin \left[\rho K^{1 / 2}\right]}{K^{1 / 2}}-2 \pi\left(\frac{\cos \left[\rho K^{1 / 2}\right]-1}{K}\right)+F \cos \left[\rho K^{1 / 2}\right]$.

When $K$ is negative (15) may more appropriately be written:

$$
\begin{aligned}
F_{\rho}= & L \frac{\sinh \left[\rho(-K)^{1 / 2}\right]}{(-K)^{1 / 2}}-2 \pi\left(\frac{\cosh \left[\rho(-K)^{1 / 2}\right]-1}{K}\right) \\
& +F \cosh \left[\rho(-K)^{1 / 2}\right] .
\end{aligned}
$$

We again note that if $K \rightarrow 0$, formulae (15) and (15') approach Steiner's result.

Observatorie de Santiago de Compostela 